\title{
Mechanical response of spiral interconnect arrays for highly stretchable electronics
}

N. Qaiser, S. M. Khan, M. Nour, M. U. Rehman, J. P. Rojas, and M. M. Hussain

Citation: Appl. Phys. Lett. 111, 214102 (2017);

View online: https://doi.org/10.1063/1.5007111

View Table of Contents: http://aip.scitation.org/toc/apl/111/21

Published by the American Institute of Physics

\section{Articles you may be interested in}

Electronic control of linear-to-circular polarization conversion using a reconfigurable metasurface Applied Physics Letters 111, 214101 (2017); 10.1063/1.4998556

A robust dual-membrane dielectric elastomer actuator for large volume fluid pumping via snap-through Applied Physics Letters 111, 212901 (2017); 10.1063/1.5005982

Uniformity of cylindrical imploding underwater shockwaves at very small radii Applied Physics Letters 111, 214103 (2017); 10.1063/1.5005174

Influence of interface inhomogeneities in thin-film Schottky diodes

Applied Physics Letters 111, 213503 (2017); 10.1063/1.5004247

Mechanically tunable integrated beamsplitters on a flexible polymer platform Applied Physics Letters 111, 211106 (2017); 10.1063/1.4998299

Transparent indium-tin oxide/indium-gallium-zinc oxide Schottky diodes formed by gradient oxygen doping Applied Physics Letters 111, 212103 (2017); 10.1063/1.4993430

\section{Scilight} Sharp, quick summaries illuminating the latest physics research

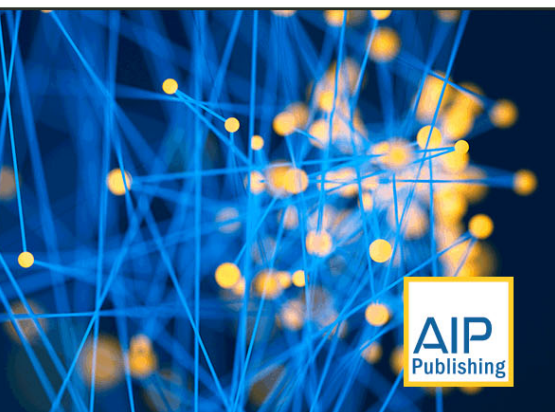




\title{
Mechanical response of spiral interconnect arrays for highly stretchable electronics
}

\author{
N. Qaiser, ${ }^{1}$ S. M. Khan, ${ }^{1}$ M. Nour, ${ }^{1}$ M. U. Rehman, ${ }^{2}$ J. P. Rojas,${ }^{2}$ and M. M. Hussain ${ }^{1, a)}$ \\ ${ }^{1}$ Integrated Nanotechnology Lab and Integrated Disruptive Electronic Applications (IDEA) Lab, \\ Electrical Engineering, Computer Electrical and Mathematical Science and Engineering Division, \\ King Abdullah University of Science and Technology (KAUST), Thuwal 23955, Saudi Arabia \\ ${ }^{2}$ Electrical Engineering, King Fahd University of Petroleum and Minerals, Dhahran 31261, Saudi Arabia
}

(Received 30 September 2017; accepted 8 November 2017; published online 21 November 2017)

\begin{abstract}
A spiral interconnect array is a commonly used architecture for stretchable electronics, which accommodates large deformations during stretching. Here, we show the effect of different geometrical morphologies on the deformation behavior of the spiral island network. We use numerical modeling to calculate the stresses and strains in the spiral interconnects under the prescribed displacement of $1000 \mu \mathrm{m}$. Our result shows that spiral arm elongation depends on the angular position of that particular spiral in the array. We also introduce the concept of a unit-cell, which fairly replicates the deformation mechanism for full complex hexagon, diamond, and square shaped arrays. The spiral interconnects which are axially connected between displaced and fixed islands attain higher stretchability and thus experience the maximum deformations. We perform tensile testing of 3D printed replica and find that experimental observations corroborate with theoretical study. Published by AIP Publishing.

https://doi.org/10.1063/1.5007111
\end{abstract}

Flexible and stretchable electronics based on various fractal designs offer great potential for a wide variety of applications ranging from bio-integrated and bioinspired devices, ${ }^{1-3}$ wearable photovoltaics, ${ }^{4}$ sensory robotics, ${ }^{5-7}$ wearable devices used for communication, ${ }^{8,9}$ to soft-robotics intended for surgical and clinical operations. ${ }^{10,11}$ Such high performance reconfigurable systems are realized through the mechanical deformations that in turn induce undesirable stresses/strains. Although these stretchable electronics have shown interesting utilities, the associated complex fabrication process and the mechanics involved during their everyday use are challenging and thus necessitate highly robust systems. For instance, soft robotics, skin sensors, and medical devices are expected to conform to the irregular surfaces or human skin while maintaining a high electrical performance, reliability, and integration. ${ }^{12}$

Today, ninety percent of the electronics are made with bulk monocrystalline silicon ( $\mathrm{Si}$ ). Nevertheless, $\mathrm{Si}$ is brittle and rigid, and thus, it cannot be stretched and essentially experiences crack-based failure during bending and stretching. Owing to favorable mechanical properties of polymers, polymeric transistors on plastic substrates have been the primary choice for stretchable electronics. ${ }^{13,14}$ Despite that the simple and low-cost fabrication process of the plastic substrate is a merit, its limited electron mobility and thermal instability obstruct further advances. Therefore, in the quest for enhanced electron mobility, the carbon based 1D and 2D materials along with graphene are under study. ${ }^{15,16}$ However, their overall performance and manufacturability are yet to be improved significantly. Therefore, Si-based compliant systems offer an optimum solution that accommodates large deformation while maintaining electronic functionalities.

\footnotetext{
${ }^{a)}$ E-mail: muhammadmustafa.hussain@kaust.edu.sa. Telephone: +966-2808-4450
}

Generally, a material with lower Young's modulus could be regarded as a compliant material. However, stiffness (higher the stiffness means harder to bend/stretch) is not described solely by the elastic modulus. But it also depends on the feature size since it carries a unit of length (i.e., $N / m$ ). Therefore, one of the successful strategies is to make the Si thinner up to a few microns, which ensures the induced strains to remain within the elastic limit. ${ }^{17}$ In the past, we have extensively reported on the CMOS compatible integration strategy for high performance flexible Si devices by reducing their thickness up to the micron level. ${ }^{18-22}$ Similarly, a stretchable wavy profile via a pre-strained polydimethylsiloxane (PDMS) sheet was attained by using an ultra-thin Si sheet which accommodated the large deformations during its operation. ${ }^{23,24}$

Flexibility and stretchability can be further enhanced by using distinctive structures such as thin island-bridge design. Islands are localized regions that hold all of the active components, i.e., sensors and actuators, whereas thin and deformable interconnects accommodate the large strains during stretching. Island-interconnect designs cover a range of structures including straight, ${ }^{25}$ curvilinear, ${ }^{26}$ free-standing designs, ${ }^{27}$ and fractal based interconnects. ${ }^{28,29}$ Rojas et al. have fabricated the Si network based on an ultra-stretchable free-standing spiral-island structure which shows excellent stretchability up to $\sim 1000 \%$ for a single spiral. ${ }^{18}$ Likewise, Rehman et al. have studied the spiral based interconnects and demonstrated the benefits of up to $\sim 55 \%$ stress reduction by replacing the straight arms with optimized serpentine ones, using numerical simulations. ${ }^{30}$ To date, most of the Finite Element Methods (FEMs) or analytical based works cover the single spiral-island system, ${ }^{18}$ as shown in the supplementary material of Fig. S1(a). However, in order to acquire the full conceptual and functional design and to attain optimum performance out of these designs, deformation behavior of spirals, which connect with islands to form 
different arrays, i.e., hexagon-arrays, as shown in Fig. S1(b) of the supplementary material, needs to be studied.

Here, we demonstrate the numerical and experimental analysis for spiral-island arrays fashioned into different geometrical morphologies. These arrays include a hexagon, diamond, square, and square with cross-linked-spirals. We have used a concept of a unit-cell in order to study the spirals connected in complex geometrical morphologies. Our results show that maximum von Mises stresses reduce for a full-array architecture as compared to widely reported single-spiral architecture while the same displacement is prescribed. For instance, maximum von Mises stress of single spiral-island reduces from $\sim 1120 \mathrm{MPa}$ to $\sim 700 \mathrm{MPa}$ when spiral arms are connected as full hexagon-array and subjected to the displacement of $1000 \mu \mathrm{m}$. Additionally, spirals that linearly connect between fixed and loaded-island attain maximum stretchability. Remaining spiral interconnects experience smaller elongations depending on spiral arm's orientation in the array. Lastly, under in-plane stretching, square design with the cross-linked spiral exhibits better performance by improving its mechanical reliability.

It is well known that in order to avoid the fracture during stretching of spiral interconnects, induced strain should reach a maximum of $1 \%$. For the maximum allowable strain $\left(\varepsilon_{\max }\right)$ of $1 \%$, the arm's breadth $\left(b_{\text {arm }}\right)$ should be 50 times thinner than the radius of the inner circle of spiral $(D)$, as governed by following relation: ${ }^{18}$

$$
\varepsilon_{\max }=\frac{b_{\text {arm }}}{D-b_{\text {arm }}} .
$$

We propose a pattern that contains the single-turn spiral with $D$ and $b_{\text {arm }}$ as $500 \mu \mathrm{m}$ and $5 \mu \mathrm{m}$, respectively, as shown in Fig. S1(a) of the supplementary material. The area near to the arm's end (towards edge of island) comprises few serpentines, which essentially add mechanical robustness. Regarding these serpentines, the radius of half-circle of serpentine $(r)$, thickness $(t)$, arch angle $(\alpha)$, and length between the half circles $(l)$ were taken as $40 \mu \mathrm{m}, 5 \mu \mathrm{m}, 45^{\circ}$, and $0 \mu \mathrm{m}$, respectively. For further details of serpentine structures, readers may refer to previous studies. ${ }^{30,31} \mathrm{Next}$, in order to account for the effect of spiral's angular orientation on the deformation behavior, we connect spirals to islands in such a manner that the final configuration mimics the shape of hexagon, diamond, and square. As the islands nearly remain stress-free during stretching, the size of islands may not contribute towards deformation behavior of spirals. ${ }^{18}$ In our study, the dimensions and shape of islands depend on the type of array under investigation, i.e., for hexagon-array; the distance between edge to edge, as shown in Fig. S1(a) of the supplementary material, was taken as $1384 \mu \mathrm{m}$. For identification purposes, we have numbered the spirals from $S_{1}$ to $S_{12}$ [supplementary material, Fig. S1(b)] in the anti-clockwise direction unless otherwise stated.

FEM (Finite Element Method) modeling was used to map the elongations and induced stresses/strains in a structure when subjected to displacement. We used a commercial FEM program ABAQUS ${ }^{\mathrm{TM}}$ to analyze the deformation behavior of spirals. All of the designs were made by using SOLIDWORKS ${ }^{\mathrm{TM}}$ and then extruded to a thickness of $75 \mu \mathrm{m}$ before importing into ABAQUS ${ }^{\mathrm{TM}}$ as $3 \mathrm{D}$ solid parts. Experimental validation of numerically simulated deformations was executed with 3Dprinted macro sized ( $\sim \mathrm{cm}$ scale) PLA (Polylactic Acid) specimens. These 3D printed spirals and islands were then arranged in various geometrical morphologies including hexagon, diamond, square, and square with a cross-linked spiral. These specimens were then stretched axially by using the experimental displacement-control tensile testing setup. As the size of specimens was scaled up to $\sim 100$ times, the prescribed displacement was taken in the range of $0 \mathrm{~cm}$ to $10 \mathrm{~cm}$. Please refer to the supplementary material for details of $3 \mathrm{D}$ printing and tensile testing.

To understand the deformation mechanism for a complex spiral-island configuration, it would be logical to start from fundamental design, i.e., single spiral, which connects between two islands [Fig. 1(a)]. Next, we add another set of spiral-islands in series as shown in Fig. 1(b). Because of spiral's thin dimension and distinctive stretchable design, spirals experience the key deformations during stretching. As a result, the islands remain stress-free during stretching. ${ }^{18}$ When we prescribe a displacement of $1000 \mu \mathrm{m}$ at the rightmost island, the spiral attached between two islands experiences the deformations during elongation, which in turn induces the stresses in the spiral. Figure 1(a) demonstrates that maximum 3D von Mises stress $\left(\sigma_{\max }\right)$ for the single-spiral case reaches up to $\sim 1120 \mathrm{MPa}$. Stretchability at the onset of maximum allowable strain (1\%) is defined as maximum stretchability $\left(E_{\max }\right)$, which is $630 \%$ for the single spiral system. However, when we add another spiral in series, i.e., called double spiral system, the prescribed displacement is now shared between both of the spirals connected in series. Figure 1(b) demonstrates that $\sigma_{\max }$ in spirals decreases from $\sim 1120 \mathrm{MPa}$ to $\sim 700 \mathrm{MPa}$ (decreases by $\sim 38 \%$ ) and the reason for stress reduction could be attributed to the smaller amount of elongations in each of the spirals. Likewise, as stretchability is a function of evolved deformations, the smaller elongations lead to reduced $E_{\max }$, i.e., $450 \%$. To validate our hypothesis, we extend the number of spirals up to four spirals that connect in series between five islands [supplementary material of Figs. S2(a) and S2(b)] and results are consistent with the double spiral system. Numerical calculations reveal that the total number of spirals connected in series shares the prescribed displacement. Figures 1(c) and 1(d) compare the evolution of stress and strain (\%) for single and double spirals systems. For fair comparison between both the designs, we define the normalized stretch as actual displacement/maximum prescribed displacement. As Si spirals experience the elastic deformations only, the results reveal that von Mises stress and strain (\%) are linearly proportional to prescribed displacement, as shown in Figs. 1(c) and 1(d).

Next, we connect multiple spiral-islands in such a fashion that the final shape of array depicts a hexagon-array as shown in the supplementary material of Figs. S1(b) and S3. It is worthy to mention that to mimic different geometrical morphologies, the shape of the island varies to accommodate the spirals that connect to island edges. For instance, twelve spirals in the full hexagon-array are represented as $S_{1}$ to $S_{12}$ and design of each island has six-edges to replicate the hexagon-array. The amount of elongation in each spiral depends on the orientation of that particular spiral in the 
(a)
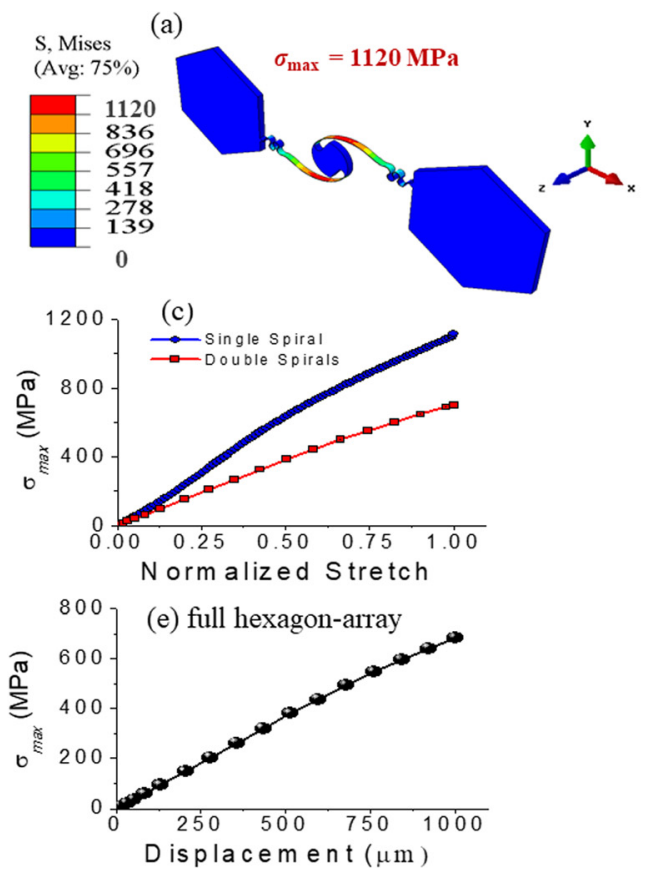

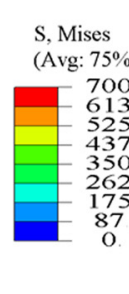

(b)
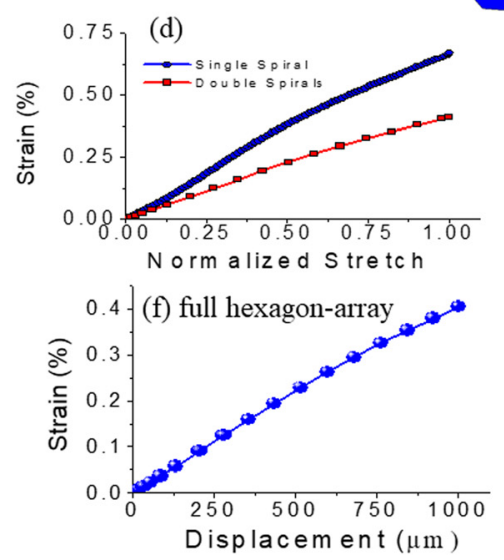

FIG. 1. Stretchability and stress distribution after connecting more spiral-islands. (a) and (b) 3D stress distribution under the same applied displacement of $1000 \mu \mathrm{m}$; calculations reveal that stretchability decreases up to $\sim 38 \%$, when additional spiral-island is connected and thus stress also reduces. (c) Maximum von Mises stress and (d) strain distributions for single and double spirals show that stress/strains are linearly proportional to normalized stretch, i.e., actual displacement/maximum prescribed displacement. (e) and (f) Maximum strain and stress as a linear function of prescribed displacement for the full-hexagon-array that consists of twelve spirals and seven islands. hexagon-array. The left-most island, i.e., island connected to spirals $\mathrm{S}_{3}, \mathrm{~S}_{4}$, and $\mathrm{S}_{10}$, shows zero prescribed displacement since it is fixed, whereas the right-most island has a maximum prescribed displacement of $1000 \mu \mathrm{m}$ as shown in the supplementary material of Fig. S3(a). These deformations lead to evolution of von Mises stresses as shown in the supplementary material of Fig. S3(b). As revealed by 3D stress distributions [supplementary material of Fig. S3(b)], each spiral has different amounts of stress, which also depends on orientation of that particular spiral. However, it is interesting to note that spirals which remain along the direction of prescribed displacement (i.e., $\mathrm{S}_{7}$ and $\mathrm{S}_{10}$ ) experience the maximum elongations and von Mises stress ( $696 \mathrm{MPa})$. We take the strain and von Mises stress at a node where it elevates to its maximum value and show these distributions as a function of prescribed displacement [Figs. 1(e) and 1(f)]. For the full hexagon-array, stress and strains are linearly proportional to prescribed displacement and are consistent with preceding single/double spiral systems.

Although numerical modeling for the full hexagon-array showed interesting results, conducting a FEM study for the full array is difficult and time-exhaustive, and results for each spiral are difficult to comprehend. Therefore, we introduce here a concept of a unit-cell. A careful examination of each array shows that array is nothing but a repetition of these unitcells. For instance, the hexagon-array can be divided into four unit-cells and each unit cell consists of three spirals and three islands, respectively, as shown in Fig. 2(a). We conducted the numerical simulation for the unit-cell of the hexagon-array, and 3D stress distribution is shown in Fig. 2(b). The $\sigma_{\max }$ for the unit cell of hexagon reaches up to $\sim 1130 \mathrm{MPa}$.

To exhibit the deformation behavior of each of the spirals in a unit-cell, the stretchability and elongations are also calculated and are shown in Fig. 2(b). During the stretching process, spirals start to unwrap and the elongation or separation between any two islands increases. In the case of the unit-cell, some spirals might experience two directional (in plane) stretch. Therefore, the distance between the centers of the islands, connected by those spirals, was regarded as the elongation. We describe this elongation as $\mathrm{dS}_{i}$ (where $i$ represents the spiral number) and then calculated the associated stretchability, i.e., $\mathrm{ES}_{i}$. Contrary to the previous configuration where all spirals were connected in series, spirals (i.e., $S_{2}$ and $\mathrm{S}_{3}$ ) in the unit cell of the hexagon-array are connected at different angular positions. Therefore, $S_{2}$ and $S_{3}$ unwrap at different rates, which results in different values of elongations, i.e., $\mathrm{dS}_{2}$ and $\mathrm{dS}_{3}$. However, as $\mathrm{S}_{1}$ is axially connected (with no angular orientation) between loaded and fixed-islands, its elongation $\left(\mathrm{dS}_{1}\right)$ is identical to the prescribed displacement. Moreover, it is interesting to note that $S_{2}$ and $S_{3}$ share the prescribed displacement, i.e., the sum of $\mathrm{dS}_{2}$ and $\mathrm{dS}_{3}$ is nearly equivalent to $\mathrm{dS}_{1}$. Likewise, stretchability holds the same relation, i.e., the sum of $\mathrm{ES}_{2}$ and $\mathrm{ES}_{3}$ is identical to $\mathrm{ES}_{1}$. To corroborate the concept of the unit-cell, we connect two unitcells, which comprise five spirals and four islands, as shown in Fig. 2(c). Deformation behavior of each of the spirals
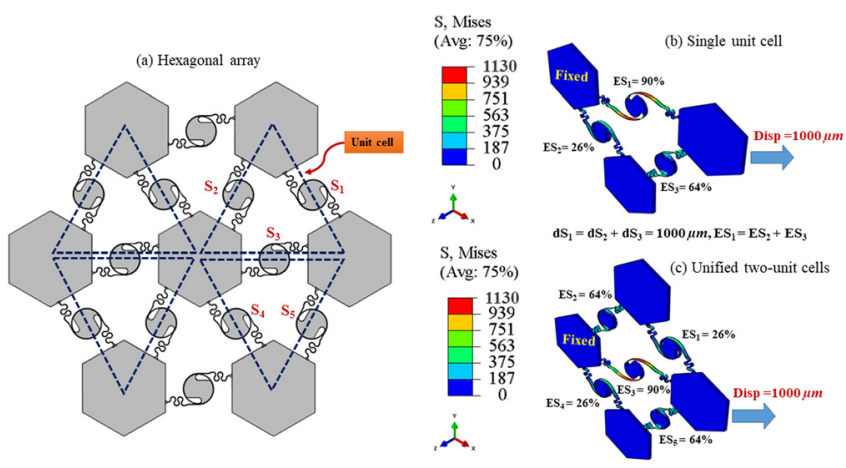

FIG. 2. (a) Deformation behavior of the full hexagon-array by utilizing the simplified concept of the unit cell. Von Mises stress distributions and stretchability of each spiral for (b) single unit cell and (c) unified two-unit cells. Spirals (i.e., $\mathrm{S}_{1}$ for the single unit cell and $\mathrm{S}_{3}$ for unified two-unit cells) which are attached directly between fixed and under-load islands experience maximum deformation $\left(\mathrm{dS}_{1}\right)$ and stretchability $\left(\mathrm{ES}_{1}\right)$. Deformations of remaining spirals follow a particular pattern but are not stretched up to their possible extent. 


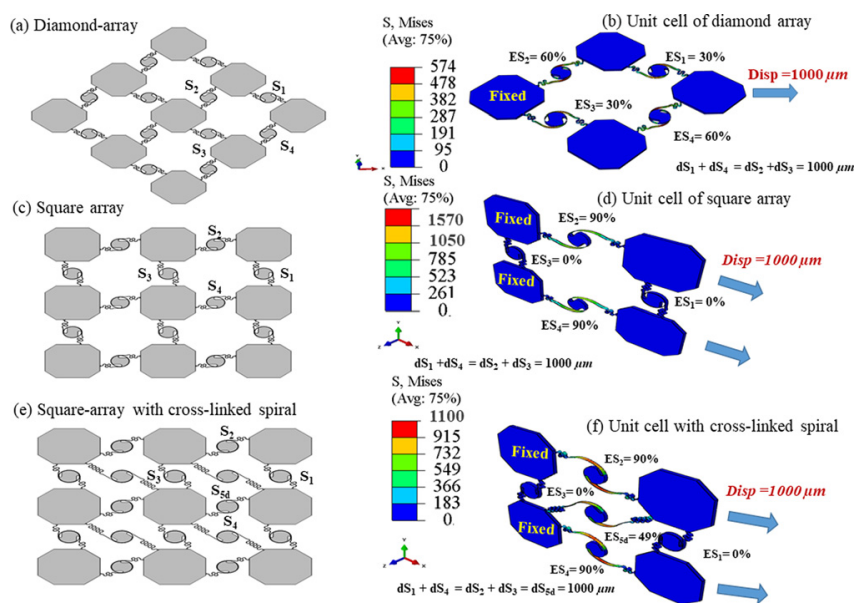

FIG. 3. Comparison of arrays shaped in different geometrical morphologies. (a) and (c) Full array representation for diamond and square. (b) and (d) Stress distribution and stretchability for each of the spirals in a respective unit cell. Square-array has more spirals that achieve maximum stretchability $\left(\mathrm{ES}_{2}\right.$ and $\left.\mathrm{ES}_{4}\right)$ when stretched along the in-plane $\mathrm{x}$-axis. Cross-linked spiral has advantage of filling the unused space, improved connectivity, and robustness. (e) Square-array with the cross-linked spiral, i.e., $S_{5 \mathrm{~d}}$, and (f) stress distribution and stretchability for each of the spirals in a unit cell for square-array with the cross-linked spiral.

$\left(\mathrm{dS}_{1}+\mathrm{dS}_{2}=\mathrm{dS}_{4}+\mathrm{dS}_{5}=\mathrm{dS}_{3}\right)$ is identical to a single unit-cell case, which evidences the validation of our unit-cell concept.

Figures 3(a) and 3(c) show the full arrays of unit cells, which mimic the diamond-array and square-array. The spiral numbering from $S_{1}$ to $S_{4}$ represents the unit-cell configuration. The von Mises stress, elongation, and stretchability for the unit cell of diamond and square-array are shown in Figs. $3(\mathrm{~b})$ and 3(d). The prescribed boundary conditions are also shown in the same figure. As expected, the angles of spirals attached with islands are different as compared to the hexagon-array, and thus, amounts of elongations also change. However, the relationship of deformations holds, i.e., $\mathrm{dS}_{1}+\mathrm{dS}_{4}=\mathrm{dS}_{2}+\mathrm{dS}_{3}=1000 \mu \mathrm{m}$. Since there is no spiral connected axially between loaded and fixed-island in the case of diamond-array, each spiral exhibits smaller elongation compared to the prescribed displacement.

For the square-array [Fig. 3(d)], $\mathrm{S}_{2}$ and $\mathrm{S}_{4}$ undergo maximum elongation and thus evolve higher stresses (up to $\sim 1570 \mathrm{MPa}$ ) as compared to hexagon and diamond-arrays. On the other hand, spirals which are along the y-direction $\left(S_{1}\right.$ and $\left.S_{3}\right)$ do not displace or unwrap at all and thus are stress-free. Therefore, one advantage of this structure could be their comparable stretchability along both the axes, i.e., $x$ and y-axes. In other words, this configuration can show better mechanical reliability when stretched in-plane along both the directions simultaneously, i.e., $\mathrm{x}$-axis and $\mathrm{y}$-axis.

Although the square-array is expected to accommodate the deformations that occur along both directions, i.e., $\mathrm{x}$-axis and $y$-axis, it has lower efficiency in terms of areal density as they have wider empty spaces in between spiral and islands. To rectify this downside, we propose a square-array and its unit cell, which consist of cross-linked or diagonally connected spiral $\left(\mathrm{S}_{5 \mathrm{~d}}\right)$, as shown in Figs. 3(e) and 3(f). The $\sigma_{\max }$ for the unit-cell decreases from $\sim 1570 \mathrm{MPa}$ to $\sim 1100 \mathrm{MPa}$. The different stresses for square and square with the crosslinked spiral are mainly due to different forces required to displace $1000 \mu \mathrm{m}$. Moreover, the cross-linked spiral provides the benefit of lower chances of failure since $S_{5 d}$ assists the additional connection, which diagonally improves connectivity between islands. $\mathrm{S}_{5 \mathrm{~d}}$ also deforms up to fully prescribed displacement of $1000 \mu \mathrm{m}$, whereas the other spiral's behavior is consistent with the previously mentioned relation, i.e., $\mathrm{dS}_{1}+\mathrm{dS}_{4}=\mathrm{dS}_{2}+\mathrm{dS}_{3}=\mathrm{dS}_{5 \mathrm{~d}}=1000 \mu \mathrm{m}$.

The comparison of FEM and experimental results is shown in Fig. 4. The unit cells of hexagon, diamond, square, and square with the cross-linked spiral were printed by using the Poly Lactic Acid (PLA) filament. The design of printed spiral including the thin dimension provides the required stretchability during tensile testing. Figures 4(a)-4(d) show the unit cells of hexagon, diamond, square, and square with the cross-linked spiral, before and after the specimens are stretched up to $\sim 10 \mathrm{~cm}$, respectively. The width and thickness of each spiral are $0.5 \mathrm{~mm}$ and $7.5 \mathrm{~mm}$, respectively. The main purpose of experiments is the mapping of elongations attained by PLA spirals and comparing with the FEM results. Widlund et al. have performed the uniaxial tensile testing of 3D printed ABS (acrylonitrile butadiene styrene) stretchable ribbons. ${ }^{31}$ Although ABS is an elastic-plastic material and experiences the yielding during tensile testing, they found

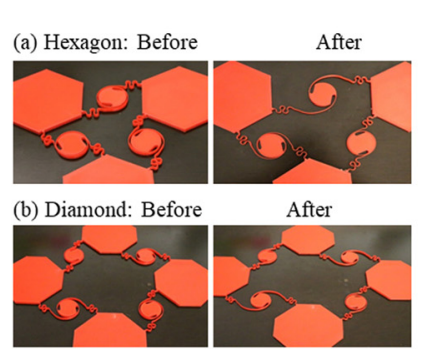

(c) Square: Before

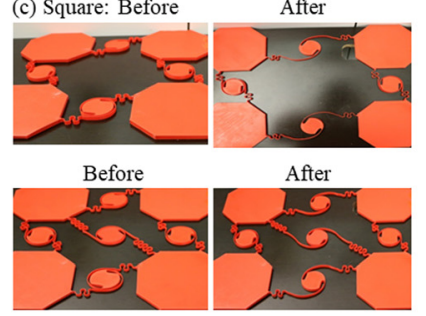

(d) Square-crosslinked (e) Hexagon

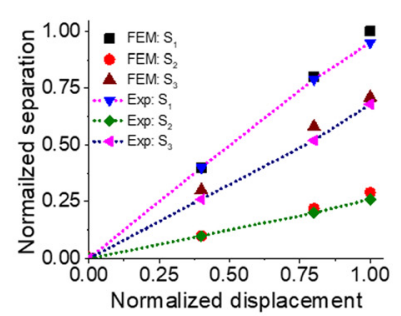

(g) Square

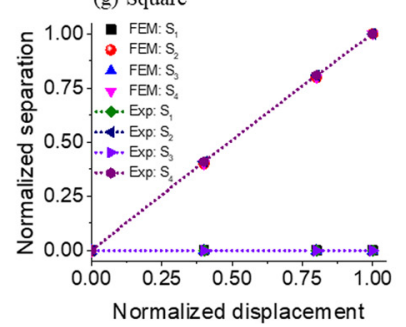

(f) Diamond

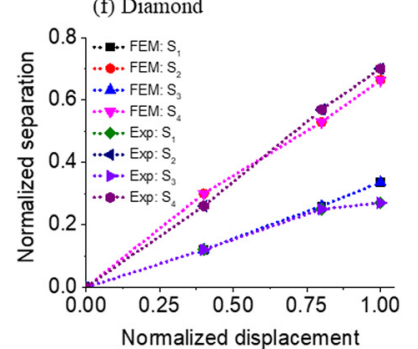

(h) Square with cross-linked

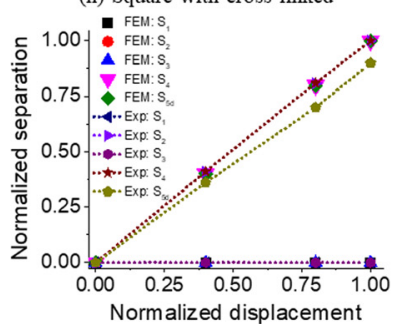

FIG. 4. 3D-printed thick unit-cells for different geometrical morphologies. Separation or elongation after stretching is measured as the distance between islands that connect the respective spiral. For fair comparison between microand macroscale specimens, normalized parameters (i.e., actual distance/maximum prescribed distance) are used. Shapes of spiral-island arrays before and after uniaxial tension testing for (a) hexagon, (b) diamond, (c) square, and (d) square with the cross-linked spiral. (e)-(h) Experimental results after testing are compared with FEM results that show that the deformation behavior of 3D-printed spirals connected as unitcells for hexagon, diamond, square, and square with cross-linked arrays is well matched with FEM calculations. 
fairly comparable strains with FEM results under a small yielding assumption. Nevertheless, our experiments use PLA, which have no yielding during tensile testing and thus measured elongation is reliable.

The results of FEM and experiments for different geometrical morphologies are shown in Figs. 4(e)-4(h), respectively. For fair comparison between micro- (prescribed displacement of $1000 \mu \mathrm{m})$ and macroscales $(\sim 10 \mathrm{~cm}$ displacement), we use normalized elongation and normalized prescribed displacement. Experimental and FEM analyses are shown in the supplementary material (SMovie 1 and SMovie 2). PLA based spiral-island unit cells stretch as expected. For instance, the sum of elongation of $S_{2}$ and $S_{3}$ is equal to $S_{1}$ in the hexagon unit cell. Likewise, the spirals in the square unit cell which are perpendicular to the load direction, i.e., $S_{1}$ and $S_{3}$, do not unwrap and their elongations are comparable with FEM results, whereas the $S_{2}$ and $S_{4}$ have experienced the maximum elongation for the prescribed displacement.

In summary, we have comprehensively studied the deformation behavior of multiple spiral-island designs. We have conducted the numerical simulations for different geometrical morphologies including hexagon, diamond, square, and square with the cross-linked array, when subjected to the prescribed displacement of $1000 \mu \mathrm{m}$. We further have introduced a concept of a unit-cell, which mimics the mechanical behavior of full complex arrays. The elongation of each spiral changes linearly with prescribed displacement and depends on spiral's position in the respective unit-cell. Our results show that stress/strains linearly change with prescribed displacement. The elongation and stress of each of the spirals reduce when more number of spirals are added and stretched under the same displacement. The square array with the cross-linked spiral has advantages of improved connectivity, areal density, and mechanical robustness by reducing the stress from $\sim 1570 \mathrm{MPa}$ to $\sim 1100 \mathrm{MPa}$. We have verified our FEM results by using the 3D printed PLA specimens. These PLA specimens were stretched with the prescribed displacement of $10 \mathrm{~cm}$. Comparison of our FEM and experimental results was found to be in good agreement with one another. The results of this study provide better understanding of spiral based stretchable interconnects which can lead to efficient designs for wearable electronics and devices.

See supplementary material for the detailed experimental and numerical procedure, schematic of the single-spiral and array of spirals, FEM analysis of four spirals connected in series, and mechanical response of the full hexagon-array.

This publication is based upon work supported by the King Abdullah University of Science and Technology (KAUST) Office of Sponsored Research (OSR) under Award No. KAUST-KFUPM Special Initiative OSR-2016-KKI2880. We declare no competing financial interest.

${ }^{1}$ S. Choi, H. Lee, R. Ghaffari, T. Hyeon, and D.-H. Kim, Adv. Mater. 28, 4203 (2016).
${ }^{2}$ H. C. Ko, M. P. Stoykovich, J. Song, V. Malyarchuk, W. M. Choi, C.-J. $\mathrm{Yu}$, J. B. Geddes III, J. Xiao, S. Wang, Y. Huang, and J. A. Rogers, Nature 454, 748 (2008).

${ }^{3}$ Y. M. Song, Y. Xie, V. Malyarchuk, J. Xiao, I. Jung, K.-J. Choi, Z. Liu, H. Park, C. Lu, R.-H. Kim, R. Li, K. B. Crozier, Y. Huang, and J. A. Rogers, Nature 497, 95 (2013).

${ }^{4}$ J. Yoon, A. J. Baca, S.-I. Park, P. Elvikis, J. B. Geddes, L. Li, R. H. Kim, J. Xiao, S. Wang, T.-H. Kim, M. J. Motala, B. Y. Ahn, E. B. Duoss, J. A. Lewis, R. G. Nuzzo, P. M. Ferreira, Y. Huang, A. Rockett, and J. A. Rogers, Nat. Mater. 7, 907 (2008).

${ }^{5}$ C. Wang, D. Hwang, Z. Yu, K. Takei, J. Park, T. Chen, B. Ma, and A. Javey, Nat. Mater. 12, 899 (2013).

${ }^{6}$ E.-S. Hwang, J. Seo, and Y.-J. Kim, J. Microelectromech. Syst. 16, 556 (2007).

${ }^{7}$ M.-Y. Cheng, C.-M. Tsao, Y.-Z. Lai, and Y.-J. Yang, Sens. Actuators, A 166, 226 (2011).

${ }^{8}$ S.-I. Park, Y. Xiong, R.-H. Kim, P. Elvikis, M. Meitl, D.-H. Kim, J. Wu, J. Yoon, C.-J. Yu, Z. Liu, Y. Huang, K. Hwang, P. Ferreira, X. Li, K. Choquette, and J. A. Rogers, Science 325(5943), 977 (2009).

${ }^{9}$ F. Axisa, P. M. Schmitt, C. Gehin, G. Delhomme, E. McAdams, and A. Dittmar, IEEE Trans. Inf. Technol. Biomed. 9, 325 (2005).

${ }^{10}$ J. Viventi, D.-H. Kim, J. D. Moss, Y.-S. Kim, J. A. Blanco, N. Annetta, A. Hicks, J. Xiao, Y. Huang, D. J. Callans, J. A. Rogers, and B. Litt, Sci. Transl. Med. 2, 24ra22 (2010).

${ }^{11}$ D.-H. Kim, N. Lu, R. Ma, Y.-S. Kim, R.-H. Kim, S. Wang, J. Wu, S. M. Won, H. Tao, A. Islam, K. J. Yu, T. Kim, R. Chowdhury, M. Ying, L. Xu, M. Li, H.-J. Chung, H. Keum, M. McCormick, P. Liu, Y.-W. Zhang, F. G. Omenetto, Y. Huang, T. Coleman, and J. A. Rogers, Science 333(6044), 838 (2011).

${ }^{12}$ J. A. Rogers, T. Someya, and Y. Huang, Science 327(5973), 1603 (2010).

${ }^{13}$ Z. Bao, Y. Feng, A. Dodabalapur, V. R. Raju, and A. J. Lovinger, Chem. Mater. 9, 1299 (1997).

${ }^{14}$ F. Garnier, R. Hajlaoui, A. Yassar, and P. Srivastava, Science 265(5179), 1684 (1994).

${ }^{15}$ S.-K. Lee, B. J. Kim, H. Jang, S. C. Yoon, C. Lee, B. H. Hong, J. A. Rogers, J. H. Cho, and J.-H. Ahn, Nano Lett. 11, 4642 (2011).

${ }^{16}$ S. J. Kang, C. Kocabas, T. Ozel, M. Shim, N. Pimparkar, M. A. Alam, S. V. Rotkin, and J. A. Rogers, Nat. Nanotechnol. 2, 230 (2007).

${ }^{17}$ J. A. Rogers, M. G. Lagally, and R. G. Nuzzo, Nature 477, 45 (2011).

${ }^{18}$ J. P. Rojas, A. Arevalo, I. G. Foulds, and M. M. Hussain, Appl. Phys. Lett. 105, 154101 (2014).

${ }^{19}$ J. P. Rojas, A. Syed, and M. M. Hussain, in 2012 IEEE 25th International Conference on Micro Electro Mechanical Systems (IEEE, 2012), pp. 281-284.

${ }^{20}$ J. P. Rojas, G. A. Torres Sevilla, M. T. Ghoneim, S. Bin Inayat, S. M. Ahmed, A. M. Hussain, and M. M. Hussain, ACS Nano 8, 1468 (2014).

${ }^{21}$ J. P. Rojas, G. A. Torres Sevilla, N. Alfaraj, M. T. Ghoneim, A. T. Kutbee, A. Sridharan, and M. M. Hussain, ACS Nano 9, 5255 (2015).

${ }^{22}$ K. Huang, R. Dinyari, G. Lanzara, J. Y. Kim, J. Feng, C. Vancura, F.-K. Chang, and P. Peumans, in 2007 IEEE International Electron Devices Meeting (IEEE, 2007), pp. 217-220.

${ }^{23}$ J. Jones, S. P. Lacour, S. Wagner, and Z. Suo, J. Vac. Sci. Technol. A 22, $1723(2004)$

${ }^{24}$ T. Li, Z. Huang, Z. Suo, S. P. Lacour, and S. Wagner, Appl. Phys. Lett. 85, 3435 (2004).

${ }^{25}$ Y. Su, J. Wu, Z. Fan, K.-C. Hwang, J. Song, Y. Huang, and J. A. Rogers, J. Mech. Phys. Solids 60, 487 (2012).

${ }^{26}$ D.-H. Kim, J. Song, W. M. Choi, H.-S. Kim, R.-H. Kim, Z. Liu, Y. Y. Huang, K.-C. Hwang, Y. Zhang, and J. A. Rogers, Proc. Natl. Acad. Sci. U.S.A. 105, 18675 (2008).

${ }^{27}$ S. Xu, Y. Zhang, L. Jia, K. E. Mathewson, K.-I. Jang, J. Kim, H. Fu, X. Huang, P. Chava, R. Wang, S. Bhole, L. Wang, Y. J. Na, Y. Guan, M. Flavin, Z. Han, Y. Huang, and J. A. Rogers, Science 344(6179), 70 (2014). ${ }^{28}$ Y. Su, S. Wang, Y. Huang, H. Luan, W. Dong, J. A. Fan, Q. Yang, J. A. Rogers, and Y. Huang, Small 11, 367 (2015).

${ }^{29}$ J. A. Fan, W.-H. Yeo, Y. Su, Y. Hattori, W. Lee, S.-Y. Jung, Y. Zhang, Z. Liu, H. Cheng, L. Falgout, M. Bajema, T. Coleman, D. Gregoire, R. J. Larsen, Y. Huang, and J. A. Rogers, Nat. Commun. 5, 3266 (2014).

${ }^{30}$ M. U. Rehman and J. P. Rojas, Extreme Mech. Lett. 15, 44 (2017).

${ }^{31}$ T. Widlund, S. Yang, Y.-Y. Hsu, and N. Lu, Int. J. Solids Struct. 51, 4026 (2014). 